\title{
PERFIL DA MORBIMORTALIDADE POR AGRESSÃO NO BRASIL: ANÁLISE DE UMA DÉCADA $_{1}$
}

\author{
Maria Nillane da Silva2 \\ Maria Camila Leal de Moura \\ Francisca Cindy de Sousa Albuquerque 4 \\ Lívia Rodrigues Abreus \\ Bruna Karinnay da Silva Sousa6 \\ Gisele Lopes Cavalcante7
}

\section{RESUMO}

A violência é grave problema mundial que repercute significativamente na vida dos indivíduos. Portanto, fazse necessário a realização de estudos acerca dos fatores relacionados à morbimortalidade por agressão, para subsidiar o enfrentamento do problema da violência no Brasil. Objetivou- se analisar o perfil da morbimortalidade por agressão no Brasil, nos últimos dez anos e corroborar com a compreensão desse agravo. Estudo documental, retrospectivo, de série temporal, realizado no mês de Agosto de 2019, baseado em dados coletados por meio do Sistema de Informações Hospitalares (SIH) do Departamento de Informática do Sistema Único de Saúde (DATASUS). Analisou- se a tendência de não completude segundo: ano do processamento, região, unidade da federação, categoria de agressão, raça/cor, faixa etária e sexo; número total de óbitos, óbitos por categoria de agressão, ano do óbito, sexo, faixa etária e valor total dos gastos hospitalares de Junho de 2010 a Junho de 2019. Constatou- se o total de 454.332 internações por agressão, com um ínfimo crescimento das taxas ano a ano sendo o ano de 2017 o que apresentou a maior taxa de internação. A região que apresentou o maior número de internações foi o Sudeste. Entre as unidades da federação, o estado de São Paulo apresentou o maior número de internações com 81.713 registros. A agressão por armas de fogo representou o maior número de internações, registrando $31,08 \%$ do total de atendimentos. A raça parda foi a mais evidente, correspondendo a 33,5\% das internações. O grupo etário de 20 a 29 anos de idade foi o mais expressivo, representando uma taxa de $30,77 \%$ do total. Em relação ao número de internações segundo o sexo da vítima, configurou- se como maioria o sexo masculino. Obtiveramse o total de 21.691 óbitos. O tipo de agressão que mais causou óbito foi por disparo de arma de fogo. Observou- se que $89,62 \%$ dos óbitos tratavam- se de pessoas do sexo masculino. A maioria dos óbitos tratouse de pessoas com 20 a 29 anos de idade. Gastou- se ao todo a quantia de R\$686.148.712,1 com as internações hospitalares por agressão. As internações por agressão no Brasil têm como protagonistas homens jovens, da raça parda, em que utiliza- se como principal instrumento de agressão a arma de fogo.

1 Trabalho apresentado no Congresso Brasileiro Ciência e Sociedade (CBCS 2019), promovido pelo Centro Universitário Santo Agostinho, de 03 a 05 de outubro de 2019, em Teresina-PI.

${ }_{2}$ Graduanda do Curso de Bacharelado em Enfermagem do Centro Universitário Santo Agostinho- UNIFSA. E-mail: marianillane12@gmail.com.

${ }_{3}$ Graduanda do Curso de Farmácia do Centro Universitário Santo Agostinho- UNIFSA. E-mail:

camilaleal.cw7@hotmail.com.

${ }_{4}$ Graduanda do Curso de Bacharelado em Enfermagem do Centro Universitário Santo Agostinho- UNIFSA. E-mail: f_cindy@outlook.com.

5 Graduanda do Curso de Bacharelado em Enfermagem do Centro Universitário Santo Agostinho- UNIFSA. E-mail: abreulivia598@gmail.com.

${ }_{6}$ Graduanda do Curso de Bacharelado em Enfermagem do Centro Universitário Santo Agostinho- UNIFSA. E-mail: karinnay@gmail.com.

7 Mestranda em Ciências Farmacêuticas pela Universidade Federal do Piauí- UFPI. E-mail:

giselecavalcante1@gmail.com.

ISBN: 978-65-80968-11-4 DOI: $10.17648 / c b c s-2019-110652$ 
ANAIS CBCS 2019 | 3 a 5 de outubro de 2019 | Centro Universitário Santo Agostinho - Teresina - PI

Sob esse viés é necessário a criação e implementação de políticas e ações de saúde voltadas a prevenção da violência nos diversos níveis de atenção, direcionando- as de acordo com as diferenças dos grupos atingidos.

Palavras-Chave: Agressão. Morbidade. Mortalidade. Sistema de informação

\section{INTRODUÇÃO}

Segundo Leite et al. (2017), a violência constitui um grave problema mundial que repercute e impacta significativamente da vida dos indivíduos. Além de refletir problemas sociais, é um grande desafio para o setor de saúde, pois consiste em um fenômeno complexo e com múltiplos fatores, dentre eles os fatores: histórico, contextuais, estruturais, culturais e interpessoais.

Assim como Melo e Garcia (2019) relatam em seu estudo, a morbimortalidade por agressão no Brasil tem como um de seus principais determinantes as desigualdades sociais, evidenciada por homicídios em contextos urbanos, cujas vítimas e agressores tratam- se em sua maioria de homens jovens. Estudos epidemiológicos evidenciam que o sexo masculino predomina entre autores e vítimas de agressões, sendo o homem geralmente agredido por outro, já a mulher por companheiro ou ex-companheiro.

De acordo com o estudo realizado por Souza et al. (2012), o indicador universal de violência social é o homicídio, definido como morte por agressão. Assim como a maioria dos agravos de saúde, as taxas de homicídio se expressam com disparidade entre países e continentes sendo a América Latina a região com a mais elevada taxa de mortalidade por homicídio, seguida pelo Caribe, África, América do Norte, Ásia, Oceania e Europa.

Conforme Malta et al. (2019), com o objetivo de desenvolver políticas públicas, programas e ações voltadas para a população jovem, em 2013, o Estatuto da Juventude incluiu entre os seus princípios e diretrizes: a promoção da vida segura e da cultura da paz, gestão da informação e produção de conhecimento (Lei no 12.852/2013).

Em virtude das características da violência no Brasil, faz-se necessário a realização de estudos acerca dos fatores relacionados à morbimortalidade por agressão principalmente no que diz respeito aos jovens e adolescentes, visto que, são altamente vulneráveis e tais estudos são 
ANAIS CBCS 2019 | 3 a 5 de outubro de 2019 | Centro Universitário Santo Agostinho - Teresina - PI

essenciais para subsidiar o enfrentamento do problema da violência no Brasil. Dessa forma, o seguinte estudo tem como objetivo analisar o perfil da morbimortalidade por agressão no Brasil, nos últimos dez anos e assim, corroborar com a compreensão desse agravo.

\section{METODOLOGIA}

Constitui-se de um estudo epidemiológico documental, descritivo, retrospectivo e de série temporal, com abordagem quantitativa e transversal, realizado no mês de Agosto de 2019, baseado em dados coletados por meio do Sistema de Informações Hospitalares (SIH/SUS) do Departamento de Informática do Sistema Único de Saúde (DATASUS). Coletaram-se dados referentes a todas as regiões federativas do Brasil.

O seguimento metodológico para determinar o critério de busca dos dados foi: acesso em Informações de Saúde, em seguida selecionou-se a opção das informações Epidemiológicas e Morbidade e na sequência Morbidade Hospitalar do SUS (SIH/SUS). Optou-se por estudar a morbidade hospitalar por causas externas por local de residência a partir do ano de 2008 nas regiões do Brasil. Dentre a estatística de morbidade, delineou-se como objeto de estudo as internações por Agressão no Brasil de Junho de 2010 a Junho de 2019, e assim, se procedeu a coleta dos dados para a elaboração dos resultados desse estudo. Analisou- se a tendência de não completude segundo as seguintes variáveis: número de internações por ano do processamento, região, unidade da federação, por categoria de agressão, raça/cor, faixa etária e sexo; número total de óbitos, por categoria de agressão, ano do óbito, sexo, faixa etária e valor total dos gastos hospitalares.

Os dados coletados foram organizados por meio de gráficos e tabelas, contabilizados como frequência absoluta e percentuais, em que se utilizou como processadores dos dados os programas: TabWin versão 4.14, Microsoft Excel versão 2016 e Microsoft Office. Por consistir em uma análise de dados secundários e de acesso público, o estudo não prevê danos ou riscos a população estudada, sendo assim, não houve a necessidade de avaliação pelo Comitê de Ética em Pesquisa (CEP) estando de acordo com a Resolução de número 466 de 12 de dezembro de 2012 do Conselho Nacional de Saúde. 


\section{conghESSO CIENCIAESOCIEDADE Inovação, Diversidatle e Sustentahilititadle}

ANAIS CBCS 2019 | 3 a 5 de outubro de 2019 | Centro Universitário Santo Agostinho - Teresina - PI

Com a intenção de aperfeiçoar a análise, prováveis hipóteses e fundamentações, realizouse um levantamento bibliográfico através das bases de dados da Biblioteca Virtual em Saúde (BVS): Literatura Latino Americana e do Caribe em Ciências da Saúde (LILACS), Scientific Eletronic Library Online (SciELO) e Medical Literature Analysis and Retrieval System Online (MEDLINE). Delimitou- se a busca com um recorte temporal de dez anos (2009-2019), utilizando- se como descritores em ciência da saúde (DECs) os termos: Agressão, Morbidade e Mortalidade, associados com o operador booleano AND.

\section{RESULTADOS E DISCUSSÃO}

Os resultados demonstraram um número total de 454.332 internações por agressão no Brasil no período estudado, com um ínfimo crescimento das taxas ano a ano sendo o ano de 2017 o que apresentou a maior taxa de internação, representando $11,52 \%$ ( $n=52.359)$ do total. Em contrapartida, o ano que apresentou o menor número de internações foi 2010, registrando apenas 25.859 atendimentos, o equivalente a 5,69\% do total como expõe a Figura 1. Consoante Trindade et al. (2015), desde o final da década de 1970 a violência no Brasil vem crescendo, principalmente o número de mortes por agressão.

FIGURA 1. Morbidade Hospitalar do SUS por agressão no Brasil no período de 2010 a 2019.

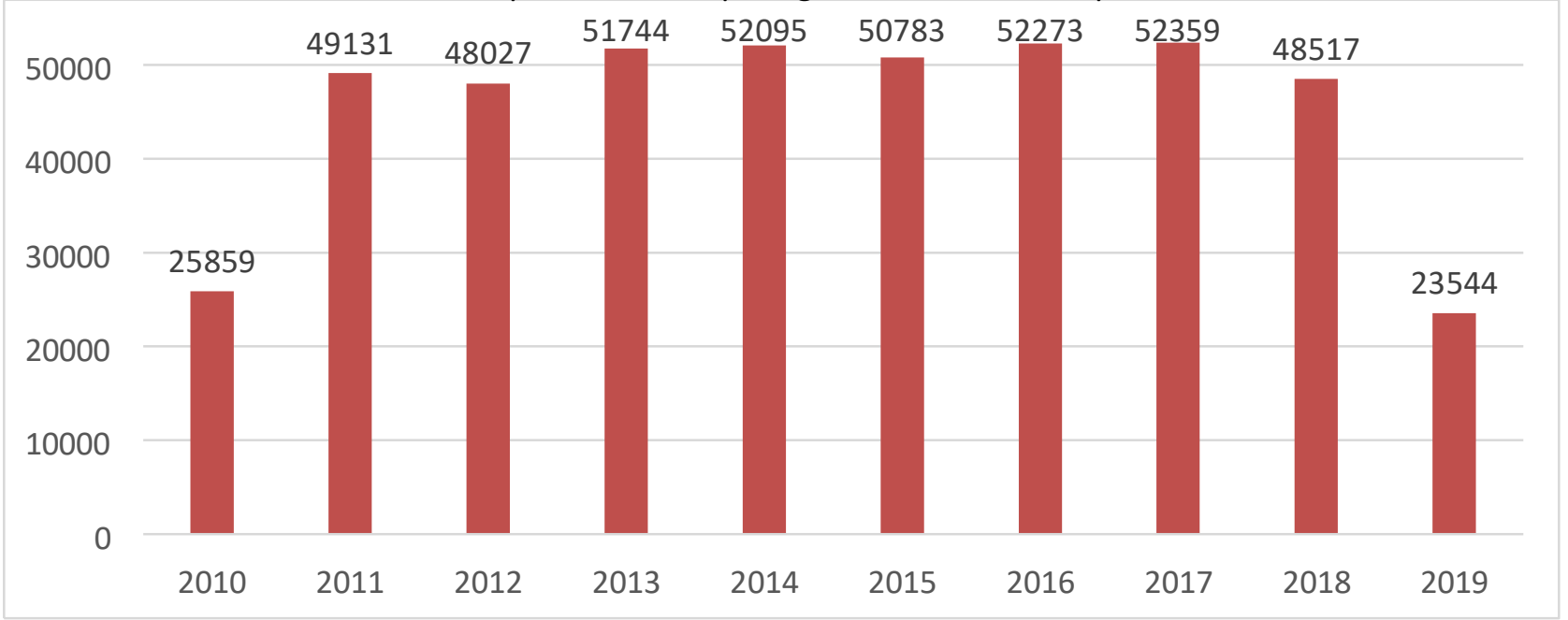

Fonte: Ministério da Saúde - Sistema de Informações Hospitalares do SUS (SIH/SUS), 2019. 


\section{CONGQEESSOCIENCIAESOCIEDADE

ANAIS CBCS 2019 | 3 a 5 de outubro de 2019 | Centro Universitário Santo Agostinho - Teresina - PI

Como mostra a Figura 1, um fato importante a ser observado é que até Junho de 2019 já foram registradas 23.544 internações por agressão, exatamente 5,18\% do total, chegando bem perto da taxa apresentada em 2010.

No que concernem as regiões do Brasil, a região que apresentou o maior número de internações foi o Sudeste com $37,27 \%$ ( $n=169.319)$ do total de internações, seguida do Nordeste com 30,61\% ( $n=139.060)$ e do Norte com 13,13\% (59.642), como mostra a Tabela 1. Entre as unidades da federação, os estados que foram destaque no número de internações foi São Paulo com 81.713 registros, seguido do estado da Bahia com 54.191 e de Minas Gerais com 54.097 atendimentos.

TABELA 1. Morbidade Hospitalar por agressão, conforme unidade da federação, Brasil, 2010 a 2019.

\begin{tabular}{lll}
\hline Região do Brasil & Frequência absoluta (n) & Frequência relativa (\%) \\
Região Norte & 59642 & 13,13 \\
Região Nordeste & 139060 & 30,61 \\
Região Sudeste & 169319 & 37,27 \\
Região Sul & 43460 & 9,57 \\
Região Centro-Oeste & 42851 & 9,43 \\
Total & 454332 & 100 \\
\hline
\end{tabular}

Fonte: Ministério da Saúde - Sistema de Informações Hospitalares do SUS (SIH/SUS), 2019.

Em consonância ao estudo de Trindade et al. (2015), a violência é maior em áreas urbanas, em que as regiões geográficas e seus estados registram um aumento da morbimortalidade por causas externas desde o ano de 1990. Acredita- se que esse fato pode estar relacionado ao maior número populacional e consequentemente à vulnerabilidade e maior exposição à ocorrência da violência, bem como a incapacidade de resistir a ela.

Em relação ao número de internações por tipo de agressão segundo categoria do CID 10 (Classificação Internacional de Doenças), a agressão por armas de fogo representou o maior número de internações, registrando $31,08 \%$ do total $(n=141.243)$ de atendimentos. Em segundo lugar, evidnciou-se a agressão por objeto cortante ou penetrante que representou o equivalente a $23,17 \%$ ( $n=105.251)$ do total. E, ainda, a agressão por meio de força corporal consolidou-se como a terceira 


\section{congenESSO CIENCIAESOCIEDADE \\ Inovação, Diversidadle e Sustentahililitadle}

ANAIS CBCS 2019 | 3 a 5 de outubro de 2019 | Centro Universitário Santo Agostinho - Teresina - PI

maior causa, registrando 82.708 atendimentos, o mesmo que $18,2 \%$ do total como evidencia a Tabela 2.

TABELA 2. Morbidade Hospitalar do SUS por agressão, conforme principais tipos, Brasil, 2010 a 2019.

Tipo de agressão

Agressão por armas de fogo

Agressão por objeto cortante ou penetrante

Agressão por meio de força corporal
31,08

23,17

82.708
18,2

Fonte: Ministério da Saúde - Sistema de Informações Hospitalares do SUS (SIH/SUS), 2019.

Conforme Minayo (2009), a arma de fogo é o principal instrumento utilizado em conflitos para produzir a morte das pessoas, principalmente entre homens. É impossível falar em homicídio no Brasil, sem fazer referência a significativa contribuição que a arma de fogo tem com a violência no país, apesar de movimentar um dos maiores mercados da economia global.

Em relação a raça/cor das vítimas de agressão (Figura 2), observou- se que a raça parda foi a mais evidente, correspondendo a 33,5\% ( $n=152.205)$ do total de internações.

FIGURA 2. Morbidade Hospitalar do SUS por agressão, segundo raça/cor, Brasil, 2010 a 2019.

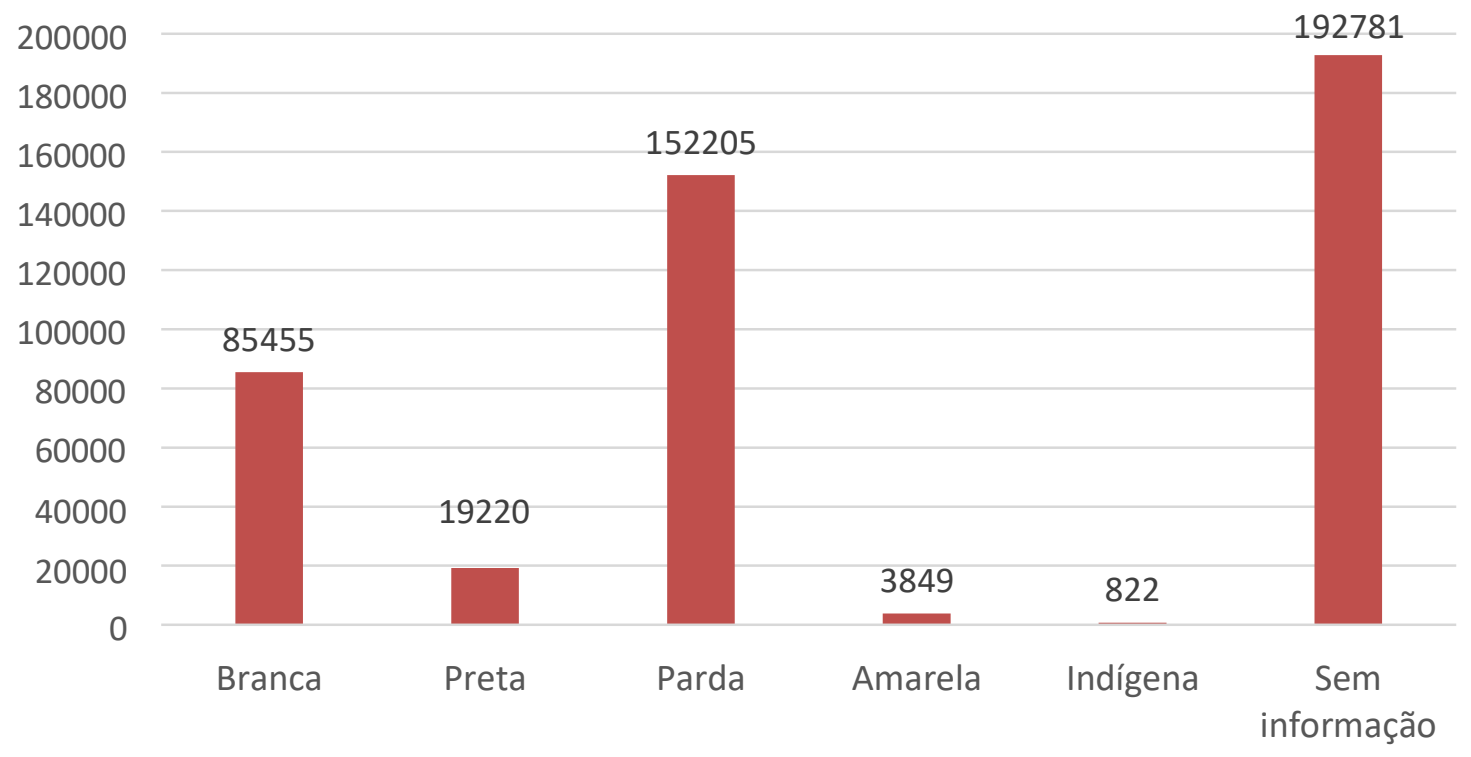

Fonte: Ministério da Saúde - Sistema de Informações Hospitalares do SUS (SIH/SUS), 2019. 


\section{conghESSO CIENCIAESOCIEDADE \\ Inovação, Diversidadle e Sustentahililitadle}

ANAIS CBCS 2019 | 3 a 5 de outubro de 2019 | Centro Universitário Santo Agostinho - Teresina - PI

De acordo com Filho (2011), a mortalidade por agressão no Brasil parace estar associada também a desigualdade racial. O número de homicídios entre negros/pardos e brancos possui diferenças significativas, mesmo controlando- se as taxas pela escolaridade do indivíduo. Isso não se deve somente ao fato de processos discriminatórios, como também de estarem em posições mais precárias no mercado de trabalho e menor índice de educação formal.

No que diz respeito à faixa etária da população agredida, teve-se que o grupo etário de 20 a 29 anos de idade foi o mais expressivo, representando uma taxa de $30,77 \%(n=139.782)$ do total de internações, como ilustra a Figura 3. O segundo grupo etário mais expressivo foi o de 30 a 39 anos com 23,1\%, seguido do grupo de 15 a 19 anos com $12,86 \%$.

Segundo Ferreira e Ribeiro (2009), a faixa etária mais atingida pelos agravantes da violência são jovens (população economicamente produtiva). Esse dado é notoriamente preocupante, visto que a alta taxa de incidência de morbimortalidade por agressão nessa população eleva as taxas de anos potenciais de vida perdidos, diminuindo a contribuição econômica e social que a vítima poderia participar futuramente. Logo, ocasiona impactos em amplas áreas como econômica, social e cultural de um país.

FIGURA 3. Morbidade Hospitalar do SUS por agressão, segundo faixa etária, Brasil, 2010 a 2019.

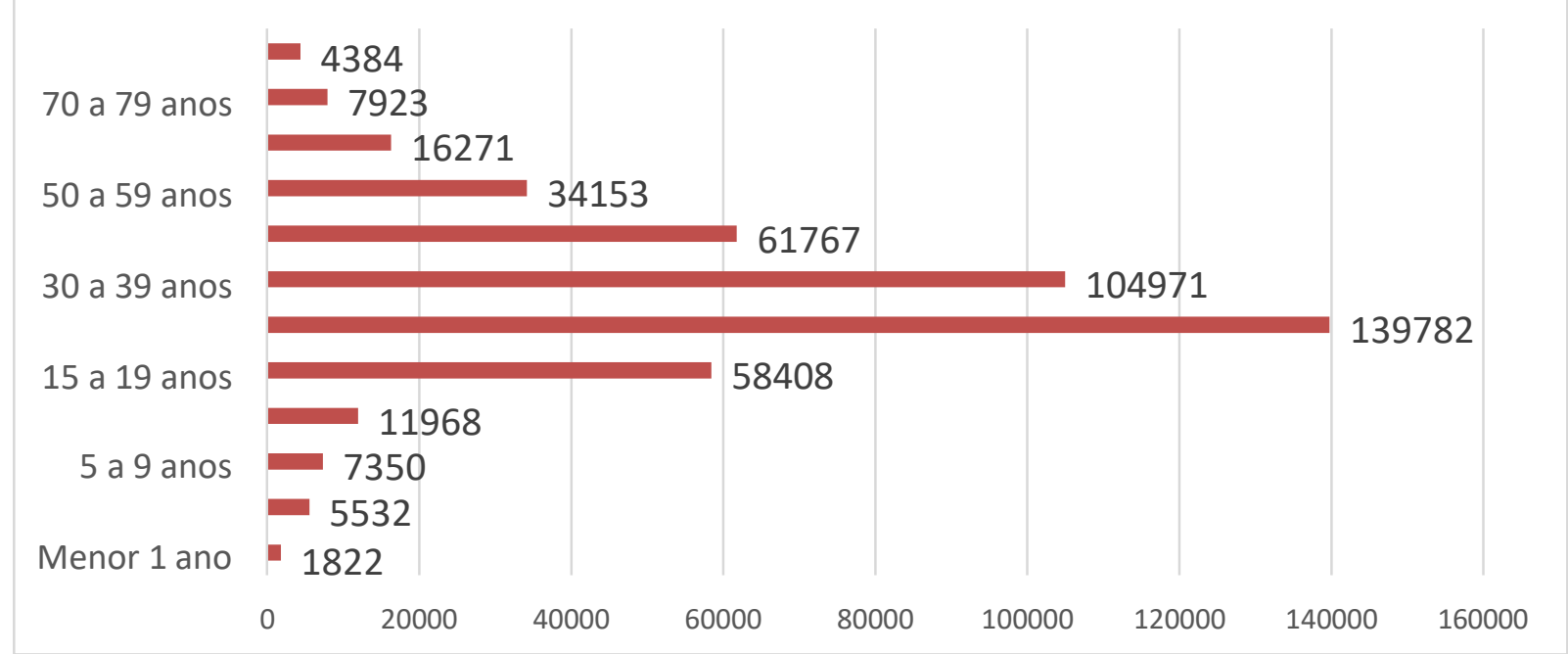

Fonte: Ministério da Saúde - Sistema de Informações Hospitalares do SUS (SIH/SUS), 2019. 


\section{conghESSO CIENCIAESOCIEDADE

ANAIS CBCS 2019 | 3 a 5 de outubro de 2019 | Centro Universitário Santo Agostinho - Teresina - PI

Em relação ao número de internações segundo o sexo da vítima, configurou- se como maioria o sexo masculino representando mais da metade das internações $(83,89 \%)$, o mesmo que 381.147 registros (Figura 4). Ferreira e Ribeiro (2009), afirmam em seu estudo que as relações de gênero na sociedade influenciam a violência e representam uma forma de afirmação da masculinidade, visto que, a maioria dos episódios violentos envolve pessoas do sexo masculino, e maioria das agressões contra mulheres são realizadas por homens, principlamente no ambiente doméstico.

FIGURA 4. Percentual de Morbidade Hospitalar do SUS por agressão, segundo sexo, Brasil, 2010 a 2019.

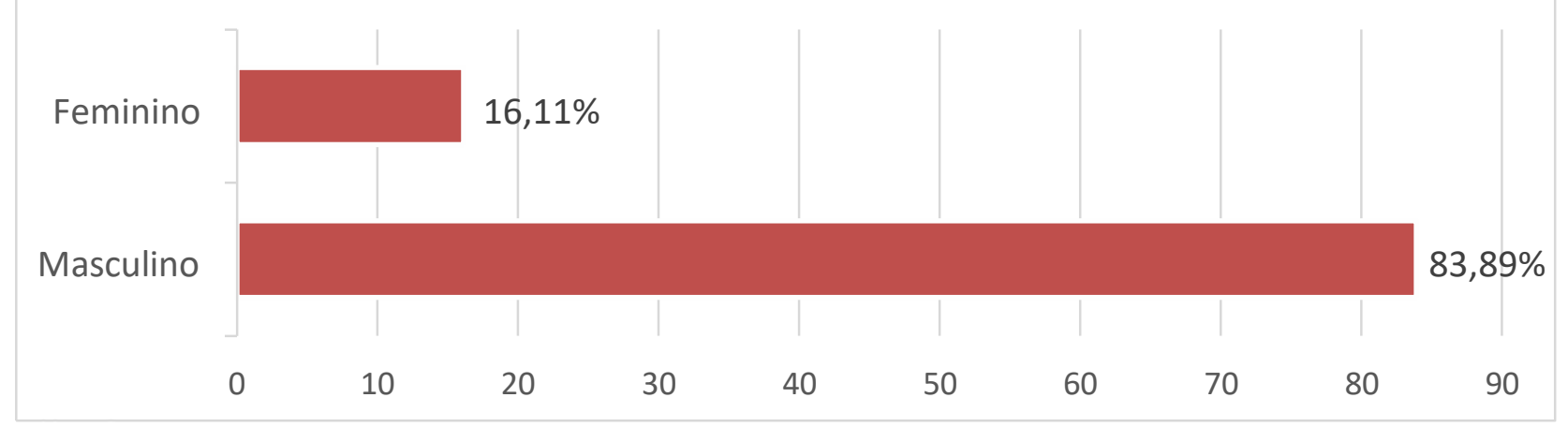

Fonte: Ministério da Saúde - Sistema de Informações Hospitalares do SUS (SIH/SUS), 2019.

A Tabela 3 demontra o número total de 21.691 óbitos por agressão, onde percebe-se uma pequena oscilação das taxas ano a ano, sendo o ano de 2014 o que mais registrou óbitos por agressão com $12,16 \%$ do total e o ano de 2010 o que menos registrou com 5,56\% no percentual.

TABELA 3. Óbitos por agressão, conforme ano, Brasil, 2010 a 2019.

\begin{tabular}{lll}
\hline Ano & Óbitos $(\mathbf{n})$ & Óbitos (\%) \\
\hline 2010 & 1206 & 5,56 \\
2011 & 2314 & 10,67 \\
2012 & 2408 & 11,1 \\
2013 & 2585 & 11,92 \\
2014 & 2638 & 12,16 \\
2015 & 2386 & 11 \\
2016 & 2570 & 11,85 \\
2017 & 2448 & 11,29
\end{tabular}




\section{congenESSO CIENCIAESOCIEDADE

ANAIS CBCS 2019 | 3 a 5 de outubro de 2019 | Centro Universitário Santo Agostinho - Teresina - PI

\begin{tabular}{lll}
2018 & 2186 & 10,08 \\
2019 & 950 & 4,38 \\
Total & 21691 & 100 \\
\hline
\end{tabular}

Fonte: Ministério da Saúde - Sistema de Informações Hospitalares do SUS (SIH/SUS), 2019.

No que tange ao tipo de agressão que mais causou óbito, teve-se o por disparo de arma de fogo com 56,61\% ( $n=956.334)$ do total de óbitos. Além disso, observou-se que $89,62 \%$ dos óbitos $(n=19.440)$ tratavam-se de pessoas do sexo masculino, e apenas $10,38 \%(n=2.251)$ do sexo feminino. Quanto ao parâmetro de número de óbitos por faixa etária evidenciou- se que a maioria dos óbitos trataram- se de pessoas com 20 a 29 anos de idade, o equivalente a $31,81 \%$ ( $n=6.899)$ dos óbitos, seguido do grupo etário de 30 a 39 anos e do grupo de 15 a 19 anos de idade, como observa- se na Figura 10. No que diz respeito ao valor total dos gastos hospitalares, gastou- se ao todo a quantia de $\mathrm{R} \$ 686.148 .712,1$ com as internações hospitalares por agressão (Tabela 4).

TABELA 4. Óbitos por agressão, conforme parâmetros de causas, sexo e faixa etária, Brasil, 2010 a 2019.

\begin{tabular}{lcc} 
Parâmetros & (n) & (\%) \\
\hline Causas & & \\
Agressão disparo de arma de fogo & 6585 & 30,36 \\
Agressão objeto cortante ou penetrante & 3293 & 15,18
\end{tabular}

\section{Sexo}

Masculino

$19440 \quad 89,62$

Feminino

2251

10,38

\section{Faixa Etária}

Menor 1 ano

104

0,48

1 a 4 anos

80

0,37

5 a 9 anos

74

0,34

10 a 14 anos

287

1,32

15 a 19 anos

2998

13,82

20 a 29 anos

6899

31,81

30 a 39 anos

4680

21,58 


\section{CONGQEESSOCIENCIAESOCIEDADE

ANAIS CBCS 2019 | 3 a 5 de outubro de 2019 | Centro Universitário Santo Agostinho - Teresina - PI

\begin{tabular}{lll}
40 a 49 anos & 2871 & 13,24 \\
50 a 59 anos & 1705 & 7,86 \\
60 a 69 anos & 984 & 4,54 \\
70 a 79 anos & 575 & 2,65 \\
80 anos e mais & 434 & 2 \\
\hline
\end{tabular}

Fonte: Ministério da Saúde - Sistema de Informações Hospitalares do SUS (SIH/SUS), 2019.

\section{CONCLUSÕES}

O estudo evidenciou que a maioria das internações por agressão no Brasil tem como protagonistas homens jovens, da raça parda, em que utiliza- se como principal instrumento de agressão a arma de fogo. O perfil da morbimortalidade por agressão no Brasil trata- se de um dado relevante para aumentar a visibilidade desse tipo de agravo.

A articulação de órgãos de segurança pública com órgãos da saúde são medidas básicas para a promoção da saúde e prevenção da violência, através da implantação da Política Nacional Para a Redução da Morbimortalidade por Causas Externas. No entanto, esse problema é de difícil resolução, pois exige uma visão multidisciplinar que componha não só ações técnicas, como também medidas políticas e socioculturais.

Sob esse viés é necessário a criação e implementação de políticas e ações de saúde voltadas a prevenção da violência nos diversos níveis de atenção, direcionando-as de acordo com as diferenças dos grupos atingidos. Ações devem ser direcionadas principalmente ao jovem do sexo masculino, no que diz respeito à violência urbana, consumo de álcool e porte de armas. Para jovens do sexo feminino essas ações devem incluir também a prevenção da violência doméstica e familiar. 


\section{cONGQESSOC CIENCIA:SOCIEDADE \\ Inovação, Diversidadle e Sustentahililitadle}

ANAIS CBCS 2019 | 3 a 5 de outubro de 2019 | Centro Universitário Santo Agostinho - Teresina - PI

\section{REFERÊNCIAS}

FERREIRA, Taciana Fernandes Araújo; RIBEIRO, Lindioneza Adriano. Epidemiologia das agressões atendidas pelo hospital de clínicas de uberlândia e dos homicídios ocorridos no município de 2000 a 2004. Bioscience Journal, v 25, n 2, p 161-170, 2009.

FILHO, Adauto Martins Soares. Vitimização por homicídios segundo características de raça no Brasil. Revista de Saúde Pública, v 45, n 4, p 745-755, 2011.

LEITE, Franciele Marabotti Costa et al. Análise da tendência da mortalidade feminina por agressão no Brasil, estados e regiões. Ciência \& Saúde Coletiva, v 22, n 9, p 2971-2978, 2017.

MALTA, Deborah Carvalho et al. Fatores associados aos episódios de agressão familiar entre adolescentes, resultados da Pesquisa Nacional de Saúde do Escolar. Ciência \& Saúde Coletiva, v 24, n 4, p 1287-1298, 2019.

MELO, Alice Cristina Medeiros; GARCIA, Leila Posenato. Fatores associados a agressões por desconhecidos entre jovens do sexo masculino atendidos em serviços de urgência e emergência: estudo de casos e controles. Ciência \& Saúde Coletiva, v 24, n 8, p 2825-2834, 2019.

MINAYO, Maria Cecília de Souza. Seis características das mortes violentas no Brasil. Revista Brasileira de Estudos Populacionais, v 26, n 1, p 135-140, 2009.

SOUZA, Edinilsa Ramos de et al. Estudo multicêntrico da mortalidade por homicídios em países da América Latina. Ciência \& Saúde Coletiva, v 17, n 12, p 3183-3193, 2012.

TRINDADE, Ruth França Cizino da et al. Mapa dos homicídios por arma de fogo: perfil das vítimas e das agressões. Revista da Escola de Enfermagem da USP, v 49, n 5, p 748-755, 2015. 